\title{
Confiabilidad intra e inter evaluador de la medición de la presión inspiratoria máxima (Pimáx) en treinta sujetos sanos de la ciudad de Cali
}

\author{
Reliability Inter and Intra Evaluator of Maximum Inspiratory Pressure (MIP) in 30 Healthy Subjects in the \\ City of Cali \\ Confiabilidade intra e inter avaliador da medição da pressão inspiratória máxima (Pimáx) em 30 sujeitos \\ sãos da cidade de Cali \\ Esther C Wilches-Luna ${ }^{1,2,3}$, Lina Marcela Sandoval MSc 1, 2, 4, David Johan López ${ }^{4}$ \\ Recibido: 26 de agosto de $2015 \bullet$ Aceptado: 5 de mayo de 2016 \\ Doi: https://dx.doi.org/10.12804/revsalud14.03.2016.02
}

Para citar este artículo: Wilches-Luna EC, Sandoval LM, López DJ. Confiabilidad intra e inter evaluador de la medición de la presión inspiratoria máxima (Pimáx) en treinta sujetos sanos de la ciudad de Cali. Rev Cienc Salud. 2016;14(3):329-338. doi: https://dx.doi.org/10.12804/revsalud14.03.2016.02

\begin{abstract}
Resumen
Introducción: La presión inspiratoria máxima (Pimáx) es un índice representativo de la fuerza global de los músculos inspiratorios. La medición de la Pimáx es un procedimiento sencillo, rápido y no invasivo, ampliamente utilizado en la práctica clínica. No existen estudios en el ámbito local que evalúen la confiabilidad de la medición de la Pimáx. Objetivo: determinar la confiabilidad intra e inter evaluador de la medición de la Pimáx. Materiales y métodos: Estudio descriptivo, de corte transversal, realizado en el marco de una prueba piloto del ensayo clínico controlado: Efectos del Entrenamiento Muscular Respiratorio en pacientes adultos en Ventilación Mecánica NCT02469064. De esta manera, 4 evaluadores realizaron 3 mediciones repetidas de Pimáx en 30 sujetos sanos. Para la evaluación de la confiabilidad intra e inter evaluador se calculó el índice de correlación intraclase (ICC) general y por cada evaluador, la concordancia entre los pares de mediciones se realizó por medio del gráfico Bland Altmant. Resultados: El Icc general fue de 0,83 (Ic95\% 0,835 - 0,939). Los valores de los Icc fueron 0,95 (Ic95 \% 0,91-0,97), 0,97 (IC95 \% 0,95-0,98),
\end{abstract}

\footnotetext{
1 Universidad del Valle. Facultad de Salud, Escuela de Rehabilitación Humana.

2 Grupo de Investigación Ejercicio y Salud Cardiopulmonar, Universidad del Valle.

3 Sociedad de Fisioterapeutas Respiratorios (Sofire S.A.S.) Unidad de Cuidado Intensivo, Clínica Farallones.

Correo electrónico: esther.wilches@correounivalle.edu.co

4 Unidad de Cuidado Intensivo Adulto, Fundación Valle del Lili.
} 
0,95 (IC95 \% 0,91-0,97), 0,91 (IC95 \% 0,85-0,97) para los evaluadores 1, 2, 3 y 4, respectivamente. El gráfico Bland Almant mostró adecuada concordancia entre las parejas de mediciones de Pimáx ( $>00,05)$. Conclusión: La Pimáx mostró ser una medida de alta confiabilidad, las mediciones no se vieron afectadas por el orden en que fueron realizadas en cada sujeto, fundamentándose así su uso en la práctica clínica.

Palabras clave: Músculos respiratorios, fuerza muscular, confiabilidad.

\section{Abstract}

Introduction: The maximum inspiratory pressure (MIP) is a representative of the overall strength of the inspiratory muscles index. Measuring the MIP is a simple, fast and non-invasive procedure widely used in clinical practice. No studies locally to assess the reliability of the measurement of the MIP. Objective: Determining the intra and inter-rater reliability of the measurement of MIP in 30 subjects Materials and methods: Descriptive study, conducted in the framework of a pilot-controlled clinical trial: Effects of Respiratory Muscle Training in adult patients on mechanical ventilation NCT02469064. Four evaluators made 3 PImax repeated measurements in 30 healthy subjects. For the evaluation of intra and inter rater reliability index intraclass correlation (ICC) general and each evaluator was calculated, the correlation between pairs of measurements were performed using Bland graphic Altmant. Results: The general ICC was 0.83 (95\% CI 0.835 to 0.939). ICC values were 0.95 (95 \% CI .91-.97), 097 (95\% CI 0.95 to 0.98), 0.95 (95\% CI from 0.91 to 0.97), 0.91 (95\% CI from 0.85 to 0.97) for evaluators 1.2, 3 and 4 respectively. The Bland Almant Figure showed adequate correlation between pairs of measurements MIP ( $\mathrm{p}>0.05)$. Conclusion: MIP proved to be a highly reliable measure, measurements were not affected by the order in which they were made for each subject, and basing its use in clinical practice.

Keywords: Respiratory muscles, muscle strength, reliability of results.

\section{Resumo}

Introdução: a pressão inspiratória máxima (Pimáx) é um índice representativo da força global dos músculos inspiratórios. A medição da Pimáx é um procedimento simples, rápido e não invasivo, amplamente utilizado na prática clínica. Não existem estudos ao nível local que avaliem a confiabilidade da medição da Pimáx. Objetivo: determinar a confiabilidade intra e inter avaliador da medição da Pimáx. Materiais e métodos: estudo descritivo, de corte transversal realizado no marco de uma prova piloto do ensaio clínico controlado: Efeitos do Treinamento Muscular Respiratório em pacientes adultos em Ventilação Mecânica NCT02469064. Quatro avaliadores realizaram 3 medições repetidas de Pimáx em 30 sujeitos sãos. Para a avaliação da confiabilidade intra e inter avaliador secalculou o índice de correlação intraclasse (ICC) geral e por cada avaliador, a concordância entre os pares de medições se realizoy através do gráfico Bland Altmant. Resultados: o ICC geral foi de 0,83 (IC 95\% 0,835 - 0,939). Os valores dos ICC foram 0.95 (IC 95\% 0.91-0.97), 0.97 (IC 95\% 0.95-0.98), 0.95 (IC 95\% 0.91-0.97), 0.91 (IC 95\% 0.85-0.97) para os avaliadores 1, 2, 3 y 4 respetivamente. O gráfico Bland Almant mostrou adequada concordância entre os pares de medições de Pimáx (p>0.05). Conclusões: a Pimáx mostrou ser uma medida de alta confiabi- 
lidade, as medições não se viram afetadas pela ordem em que foram realizadas em cada sujeito, fundamentando-se assim o seu uso na prática clínica.

Palavras-chave: Músculos respiratórios, força muscular, confiabilidade.

\section{Introducción}

La presión inspiratoria máxima (Pimáx) es un índice representativo de la fuerza global de los músculos inspiratorios (diafragma e intercostales externos como los más importantes); además de un conjunto de variables como las relaciones de longitud-tensión, frecuencia de estimulación y velocidad de contracción que presentan dichos músculos (1).

Esta prueba mide la presión (en cm $\mathrm{H} 2 \mathrm{O}$ o $\mathrm{mmHg}$ ) generada por los músculos respiratorios al realizar una maniobra inspiratoria en contra de una vía aérea ocluida. Esta medida puede ser realizada por diferentes áreas (nariz, esófago, boca y estómago). Sin embargo, la más comúnmente realizada, por su carácter no invasivo, es la medida de la presión en boca que se realiza con una boquilla especial y un adaptador al cual se conecta el transductor de presión. La medición de la Pimáx es un procedimiento ampliamente utilizado en la práctica clínica para evaluar la fuerza muscular respiratoria (1). La evaluación del desempeño muscular respiratorio es necesaria en el diagnóstico y seguimiento de la mayoría de los pacientes con enfermedades respiratorias crónicas; sin embargo, para que las pruebas de fuerza de los músculos respiratorios aporten información precisa y exacta deben realizarse de manera estandarizada, es decir, siguiendo procedimientos uniformes que minimicen las fuentes de variación (2).

En la práctica clínica es imprescindible el uso de mediciones confiables, ya que mediciones subjetivas pueden comprometer los resultados de las intervenciones.
La literatura con respecto a la estandarización de la medición de la Pimáx es creciente. En 1999, Smeltzer et al. evaluaron la fiabilidad de la Pimáx y de la Pemáx en sujetos con esclerosis múltiple (EM) y en controles sanos mediante la identificación del número de sesiones de prueba y el número de maniobras (3). Los resultados sugirieron que varias sesiones de práctica deberían indicarse para obtener valores de Pimáx y Pemáx fiables en personas con EM y al menos una sesión de práctica debía ser proporcionada a los sujetos sanos antes de identificar una línea de base.

Montemezzo et al. evaluaron la influencia de 4 interfaces diferentes en la capacidad para generar Pimax y Pemáx y el impacto de estas interfaces en la aceptabilidad y la repetitividad de las mediciones y encontraron que no hubo diferencia significativa entre las 4 interfaces, Pimáx ( $p \geq 0,49$ ) o Pemáx ( $p \geq 0,11)$, ni en el número de pruebas realizadas para cumplir los criterios de repetitividad para Pimáx $(\mathrm{p}=0,69)$ o Pemáx $(p=0,47)$ (4).

Braga et al. evaluaron la reproductibilidad test-retest y la validez concurrente del manovacuómetro digital en la medición de la Pimáx y Pemáx y de la presión inspiratoria nasal (SNIP) en 30 individuos sanos (20-30 años) y no encontraron diferencias significativas entre los valores medios obtenidos con los dos instrumentos ( $p>0,05)$ (5).

Obando et al. determinaron los valores de Pimáx y Pemáx en sujetos sanos mayores de 20 años de la zona urbana de Manizales, Colombia y los correlacionaron con variables sociodemográficas y antropométricas; sin embargo, en el estudio no se identifican datos de confiabilidad 
intra e inter evaluador de la medición de la Pimax (6).

La confiabilidad de un instrumento está relacionada con el grado en que se obtienen las mismas medidas al aplicar dos veces el mismo instrumento al mismo sujeto o grupo de sujetos, mediando entre ambas tomas en un tiempo determinado. Entre ambas tomas pueden intervenir una serie de factores que son fuentes de variabilidad y de error, lo que disminuye la confiabilidad de las medidas (7).

Todo instrumento o método de evaluación debe ser analizado con respecto a su confiabilidad, lo cual está relacionado con la capacidad del instrumento para evaluar o medir en momentos diferentes la misma medida. Un instrumento confiable mide fenómenos de forma fidedigna, secuencial, exacta y predecible, sin cambios. Si una evaluación funcional o cualquier test no es confiable, el estado inicial del paciente o el efecto del tratamiento puede verse modificado (8).

Estudios de confiabilidad en el área de medición y evaluación en fisioterapia cardiopulmonar son necesarios para asegurar que el error que pueda presentarse en las mediciones sea reducido, y para detectar cambios en lo que se está midiendo.

En el ámbito local no se conocen estudios que describan la confiabilidad intra e inter evaluador de la Pimáx medida con el manovacuómetro.

Teniendo en cuenta lo anterior, el objetivo de este estudio fue determinar la confiabilidad intra e inter evaluador de la presión inspiratoria máxima (Pimáx) en 30 sujetos sanos de la ciudad de Cali, en el periodo septiembrenoviembre de 2014

\section{Materiales y métodos}

Tipo de estudio: Descriptivo, de corte transversal, que realizó el análisis de las mediciones repetidas de Pimáx, consignadas en la base de datos de una prueba piloto del ensayo clínico controlado: "Efectos del Entrenamiento Muscular Respiratorio en pacientes adultos en Ventilación Mecánica (кст02469064)".

Población y muestra: La población estuvo conformada por 30 sujetos escogidos por conveniencia, quienes cumplieron con los criterios de inclusión y que voluntariamente participaron en la prueba piloto.

Criterios de inclusión: Personas sanas, que manifestaron de manera verbal no tener antecedentes de enfermedades pulmonares o instauradas en el momento de la medición, con edades entre los 18 y los 70 años.

Criterios de exclusión: Personas con alteraciones osteomusculares, antecedentes de cirugías abdominales, pulmonares o trastornos neurológicos, personas en las que fuese necesario realizar ajustes o variaciones para la medición de la Pimáx.

Para conservar el anonimato de los participantes y garantizar la confidencialidad de los datos se le asignó un código a cada participante que se formaba con la primera letra de cada nombre y de cada apellido. Se explicó el procedimiento de medición de Pimáx, la confidencialidad y privacidad con la información proporcionada a todos los participantes del estudio, y la privacidad del paciente, pues no sería identificado en ninguna publicación. Luego de la explicación del procedimiento, si el sujeto aceptaba participar en la prueba piloto y lo manifestaba con la firma del consentimiento informado, se realizaba la medición.

Se diseñó un formato de recolección de datos, donde se incluyeron las variables a evaluar (edad, género y Pimáx). El equipo para medición de la Pimax fue un Manovacuómetro marca Carefusión ${ }^{\circledR}$, es un manómetro digital que permite el registro de presiones en la boca en centímetros de agua $\left(\mathrm{cm}-\mathrm{H}_{2} \mathrm{O}\right)$ utilizado por diversos autores como Neder y Cader $(9,10)$. Este equipo fue 
calibrado previamente a su uso en el departamento de electromedicina de una institución de salud de cuarto nivel de la ciudad de Cali.

Se diseñó un Manual Operativo para la medición de la Pimax, fundamentado en los protocolos descritos por la American Thoracic Society (ATS) y la European Respiratory Society (ERS) (11, 12). Cada evaluador fue entrenado y capacitado previo al inicio de la prueba piloto.

Las mediciones fueron realizadas por 4 fisioterapeutas especialistas en fisioterapia cardiopulmonar. Cada uno de los evaluadores realizó 3 mediciones de Pimáx en cada sujeto sano. Las mediciones fueron realizadas durante 3 días consecutivos, en el horario de 2:00 p.m. a 6:00 p.m. El registro de la medición fue realizado por una persona externa al proyecto.

\section{Protocolo de medición}

Los participantes estaban sentados, con la cadera en un ángulo de $90^{\circ} \mathrm{y}$ los pies apoyados en el suelo. Posteriormente, cada evaluador instruía y explicaba a la persona la medición a ser realizada por medio de los siguientes comandos: "Le voy a medir la fuerza de los músculos respiratorios, para ello usted va a sostener la boquilla con sus labios". Luego se instruía para que la persona botara el aire suave (Capacidad residual funcional) y luego que inspirara tan fuerte y rápido como le fuera posible, generando un único esfuerzo inspiratorio. El evaluador ubicaba correctamente la boquilla, la nariguera y el manovacuómetro. El comando estandarizado fue el siguiente "Listo don XX bote el aire que contiene en su pulmones y ahora tómelo lo más rápido y profundo que pueda, sosténgalo 1, 2, 3, 4, 5, descanse. Se finalizaba cada medición con los siguientes comandos: "Muy bien hecho, ahora va a descansar 2 minutos, repetiremos el mismo ejercicios 2 veces más".

Para la realización de la segunda y tercera medición de la Pimáx se instruía el periodo de reposo, se retiraba la boquilla y la nariguera nuevamente a la persona con los siguientes comandos: "Va a realizar la misma maniobra tal y como lo realizó hace un rato".

Para que las mediciones fueran consideradas técnicamente aceptables, no podía haber fuga de aire audible alrededor de la boquilla detectado por el investigador o informado por el sujeto, y la presión tenía que ser mantenida durante al menos 5 segundos. Un periodo de descanso mínimo de 2 minutos fue proporcionado entre las mediciones, y entre cada evaluador se realizaba un periodo de 15 minutos de descanso. Los sujetos fueron alentados durante las mediciones para lograr los más altos valores posibles. Se realizaban 3 mediciones y se tomaba el mayor valor.

El registro de la Pimax, del tiempo entre cada medición por cada evaluador y entre evaluadores, la edad y el sexo de los sujetos fueron realizadas por una persona externa al proyecto.

Para las estrategias de control de calidad de la recolección de los datos, el investigador principal estuvo presente en el momento de las mediciones con el fin de verificar el cumplimiento del manual operativo estandarizado de medición de la Pimax. Al finalizar cada jornada de medición, el investigador principal revisaba el formato de registro de medición de la Pimax con el fin de identificar fallas en el registro de los datos y evitar sesgos de información.

Investigación con riesgo mínimo, según la Resolución 8430 de 1993, contó con el aval del Comité de Ética de la Universidad del Valle (\# 022-010,) y tuvo en cuenta las disposiciones contempladas en la Declaración Helsinky.

\section{Análisis estadístico}

Las variables sociodemográficas, edad y género de los sujetos que ingresaron al estudio se analizaron según la escala de medición de cada variable, por medio de medidas de tendencia central, dispersión, tablas de frecuencia e intervalos de confianza al $95 \%$. La evaluación de la distribución de la 
Pimáx se analizó por medio de la prueba Shapiro Wilk, una vez comprobada la normalidad, para la evaluación de la confiabilidad intra e inter evaluador de la Pimáx, se calculó el índice de correlación intraclase. Para evaluar la concordancia entre los pares de mediciones, se realizó el análisis Bland Altmant $(13,14)$. Todos los cálculos fueron realizados en el programa SPSS y Xl-stat.

\section{Resultados}

Los 4 evaluadores realizaron 3 mediciones de Pimáx en 30 sujetos sanos, en total se realiza- ron 360 mediciones. El promedio de la edad de sujetos del estudio fue 28 años (DE+/- 4), 20 sujetos eran mujeres y 10 sujetos hombres.

El valor del Icc de 0,83 Ic95 (0,83-0,93) mostró una concordancia buena entre las mediciones de Pimáx para los 4 evaluadores. Cada evaluador obtuvo valores del ICC mayores a 0,90 , indicando así una buena correlación de las 3 medidas al interior de cada evaluador (tabla 1).

La evaluación de la magnitud de la discrepancia mediante el análisis de los gráficos de Bland y Altman para las 3 parejas de medicio-

Tabla 1: Variabilidad intra-evaluador y entre-evaluadores de la Pimáx

\begin{tabular}{lccccc}
\hline & $\begin{array}{c}\text { Pimáx 1 } \\
\text { cmH20 } \\
(\text { Media/DE })\end{array}$ & $\begin{array}{c}\text { Pimáx 2 } \\
\text { cmH20 } \\
(\text { Media/DE })\end{array}$ & $\begin{array}{c}\text { Pimáx 3 } \\
\text { cmH20 } \\
(\text { Media/DE })\end{array}$ & ICC & IC95\% \\
\hline Evaluador 1 & $89,10(35,9)$ & $91,2(36,1)$ & $90,10(35,2)$ & 0,955 & $(0,919-0,977)$ \\
Evaluador 2 & $90,23(35,1)$ & $88,9(35,7)$ & $89,58(34,98)$ & 0,973 & $(0,951-0,986)$ \\
Evaluador 3 & $81,70(34,0)$ & $82,9(30,2)$ & $82,25(31,4)$ & 0,953 & $(0,916-0,976)$ \\
Evaluador 4 & $81,50(29,1)$ & $83,4(31,1)$ & $82,37(29,1)$ & 0,918 & $(0,857-0,957)$ \\
General & $85,60(34,0)$ & $86,6(33,7)$ & $86,31(33,1)$ & 0,830 & $(0,835-0,939)$ \\
\hline
\end{tabular}

Pimax: Presión inspiratoria máxima; de: Desviación Estándar; ıcc: Índice de Correlación intraclase, ıc: intervalo de confianza.

nes de Pimáx mostró que la mayor proporción de los valores de las diferencias entre parejas de mediciones de la Pimáx se encontraron dentro del intervalo de confianza, alrededor de 0 , lo que evidencia una concordancia adecuada $(\mathrm{p}>0,05)$ (Pimáx 1-Pimáx 2, p=0.46), (Pimax 2-Pimax 3, $\mathrm{p}=0.42)($ Pimax 1- Pimax3 $\mathrm{p}=0,50)$ (figura 1). 

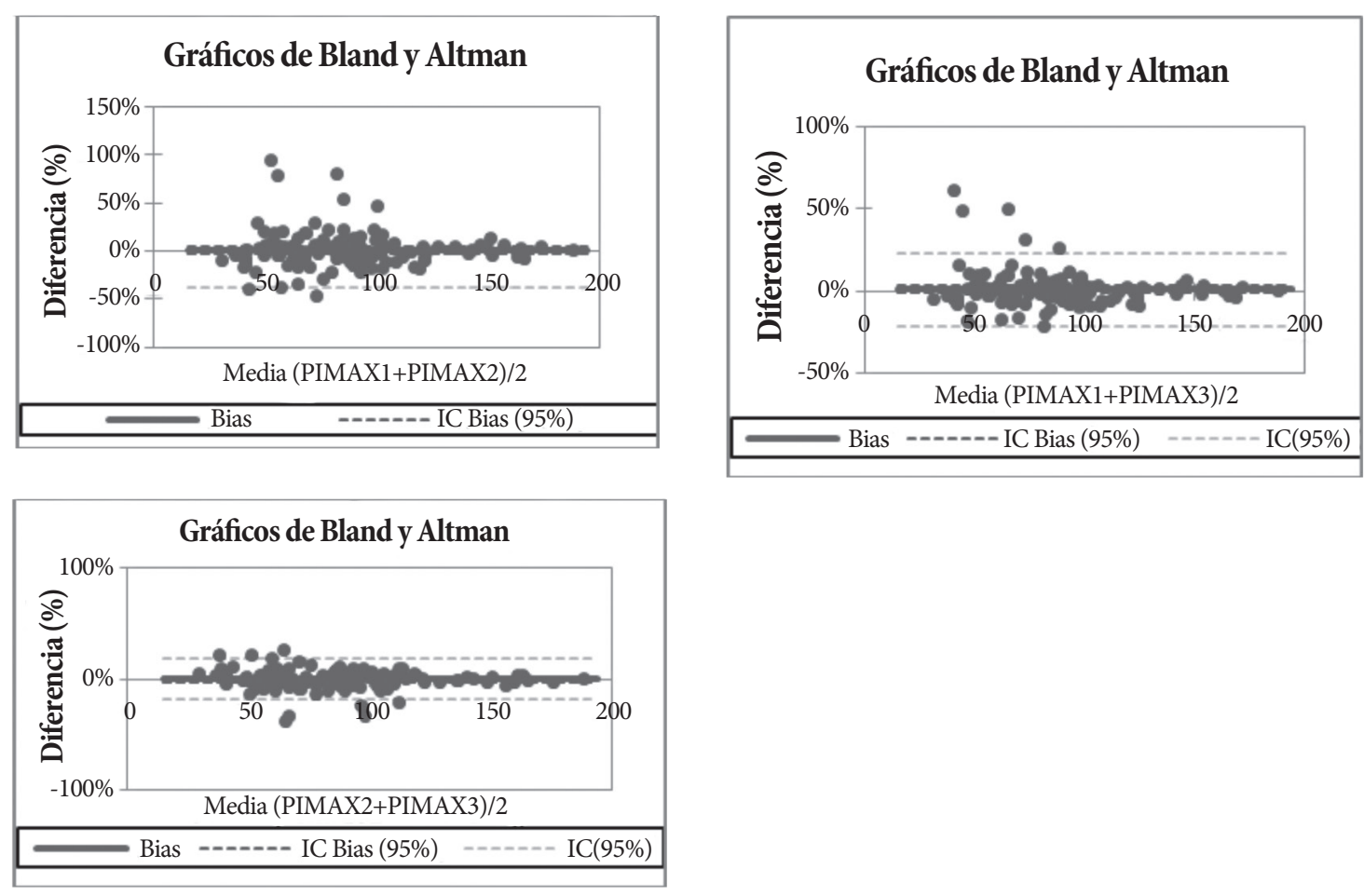

Figura 1. Gráficos de Bland y Altman para las 3 parejas de mediciones

\section{Discusión}

Los resultados del presente estudio mostraron buena confiabilidad intra e inter evaluador de las medidas de Pimáx. Pese al uso frecuente del manovacuómetro como instrumento para evaluar la fuerza global de los músculos respiratorios, en el medio son escasas las publicaciones que reporten la correspondencia entre las mediciones realizadas por diferentes evaluadores (15-17).

Los resultados mostraron un ICC de 0,86 , lo que sugiere una alta confiabilidad inter e intra evaluador en las mediciones de la Pimáx; en este estudio, además del ICC, se usó el test de concordancia de Bland y Altman, y se observó una alta concordancia y ausencia de diferencias significativas entre las parejas de mediciones de la Pimáx.

Los 4 evaluadores presentaron altos índices de confiabilidad, el evaluador $\mathrm{N}^{\circ} 1$ de 0,955 IC95 \% (0,919-0,977), el evaluador N. ${ }^{\circ} 20,973$
IC95 \% (0,951-0,986), el evaluador N. ${ }^{\circ} 30,953$

IC95 \% (0,916-0,976), y el evaluador N. ${ }^{\circ} 4$ 0,918 IC $95 \%(0,857-0.957)$, a la fecha no se identificaron estudios que evaluaran la confiabilidad de la Pimáx al interior de cada evaluador. Para los autores, la alta confiabilidad intraevaluador pudo estar relacionada con la familiaridad que tuvieron los evaluadores con el instrumento de medición y el aprendizaje adecuado de la técnica de medición.

El gráfico Bland Altmand mostró adecuada concordancia entre las parejas de mediciones, lo que indicó que no fueron afectadas por el orden en que se realizaron $(18,19)$. Investigadores sugieren que existe un efecto de aprendizaje que influye en las mediciones en un mismo sujeto, en este orden de ideas, cada medición puede ser más alta por ese aprendizaje, lo que brinda una mayor confiabilidad en las medicio- 
nes (7). En este estudio, los resultados sugieren que las mediciones no fueron afectadas por el orden en que se realizaron, los investigadores asumen que el entrenamiento realizado favoreció a una mayor confiabilidad de las mediciones.

Dimitriadis et al. reclutaron 15 estudiantes de fisioterapia de una institución educativa (9 hombres y 6 mujeres) y reportaron valores de ICC $>0,90$ para la medición de Pimáx con un manómetro digital (16). Parreira et al. en 2007 reclutaron 103 sujetos ( 47 hombres y 56 mujeres) y observaron un ICC de 0,89 para la misma medida, resultados parecidos con los obtenidos en esta investigación, en donde, en condiciones similares a las de los estudios anteriores, se encontró un ICC de 0,86 (20).

En las publicaciones revisadas no se identificó uniformidad en el número de mediciones para obtener los valores de Pimáx. Neder et al. midieron la Pimáx en 100 sujetos no fumadores (50 mujeres y 50 hombres) y realizaron entre 3 y 5 mediciones (9). Parreira et al. reportaron 3 mediciones $\mathrm{y}$, entre estas, un número mínimo de 2 reproducibles y el último valor encontrado no podía ser superior a los demás (18). En el presente estudio se realizaron 3 mediciones, siguiendo las directrices de la ATs y, al igual que Cader et al., se escogió el mejor valor (10).

El tipo de manómetro recomendado para la medición de la Pimáx es aquel capaz de cumplir con los requisitos de confiabilidad, precisión y reproductibilidad de la medida que se propone (21). Directrices recomiendan el uso del manovacuómetro digital debido a su alta precisión. Autores brasileros evaluaron la reproducibilidad test-retest y la validez concurrente de los manovacuómetros digitales UfMG y MicroRPM® (Micro Medical, UK) para medir Pimáx y Pemáx y la presión inspiratoria nasal (SNIP), y encontraron que la correlación entre los valores observados en los dos ins- trumentos fue de alta magnitud para todas las variables $(0,82$ a 0,85$)$ y no se presentaron diferencias significativas entre los valores medios obtenidos en los dos instrumentos ( $p>0,05)(5)$.

En este estudio se utilizó un manovacuómetro digital, como lo recomiendan las directrices, y aunque no se evaluó la validez del instrumento, los autores consideran que los resultados sugieren que el manovacuómetro usado es un instrumento confiable para la evaluación de la Pimáx.

La utilidad clínica que aporta este estudio adquiere un especial interés en el campo de la evaluación en fisioterapia cardiopulmonar, en donde continuamente se toman decisiones basadas en mediciones y se pone de manifiesto la importancia de la confiabilidad en la medición de la Pimax como una medida que puede ser considerada válida y confiable. Los autores reconocen que las limitaciones del estudio están relacionadas con la escasa literatura que permitiera establecer similitudes y diferencias con otros estudios.

\section{Conclusiones}

La medición de la Pimáx con el manovacuómetro digital presentó alta confiabilidad inter e intra evaluador y la concordancia de cada medición no resultó afectada por el orden en que fueron realizadas.

\section{Recomendaciones}

Se recomienda realizar estudios de concordancia y validez comparando la medición de la Pimax con técnicas que no requieran esfuerzo voluntario del paciente, como la medición de la presión transdiafragmática o con técnicas electrofisiológicas como la electromiografía y el test de estimulación Twich, entre otras.

\section{Conflicto de intereses}

Los autores declaran no tener ningún conflicto de interés en la realización del estudio. 


\section{Referencias}

1. Montemezzo D, Vieira DS, Tierra-Criollo CJ, Britto RR, Velloso M, Parreira VF. Influence of 4 interfaces in the assessment of maximal respiratory pressures. Respir Care 2012;57(3):392-8. doi: 10.4187/ respcare. 01078

2. Nici L, Donner C, Wouters E, Zuwallack R, Ambrosino N, Bourbeau J, et al. ATs/ERs Pulmonary Rehabilitation Writing Committee. American Thoracic Society/European Respiratory Society Statement on Pulmonary Rehabilitation. Am J Respir Crit Care Med 2006;173:1390-413.

3. Smeltzer SC, Lavietes MH. Reliability of maximal respiratory pressures in multiple sclerosis. Chest 1999;115(6):1546-52.

4. Dassios T, Katelari A, Doudounakis S, Mantagos S, Dimitriou G. Respiratory muscle function in patients with cystic fibrosis. Pediatr Pulmonol 2013;48(9):865-73. doi: 10.1002/ppul.22709

5. Braga Sclauser Pessoa IM, Alves Pereira HL, Tavares Aguiar L, Leite Tagliaferri T, Mendes da Silva LA, Franco Parreira V. Test-retest reliability and concurrent validity of a digital manovacuometer. Fisioter. Pesqui. 2014;21(3):236-42. doi: 10.590/1809-2950/63521032014.

6. Gil Obando LM, López López A, Ávila CL Normal values of the maximal respiratory pressures in healthy people older than 20 years old in the City of Manizales-Colombia. Colombia Médica 2012;43(2):119-25.

7. Meliá JL. La Construcción de la Psicometría como Ciencia Teórica y Aplicada. Valencia: Cristóbal Serrano; 1990.

8. Cardoso J, Azevedo N, Cassano C, Kawano M, Âmbar G. Intra and inter observer reliability of angular kinematic analysis of the hip joint during the sit-and-reach test to measure hamstring length in university students. Rev Bras Fisioter 2007;11(2):133-8.

9. Neder JA, Andreoni S, Lerario M, Nery L. Reference values for lung function tests: II. Maximal respiratory pressures and voluntary ventilation. Braz J Med Biol Res 1999;32(6):719-27.

10. Cader S, Bezerra da Silva E, Vale R, Bacelar S, Monteiro MD, Dantas E. El efecto del entrenamiento de los músculos inspiratorios en la presión inspiratoria máxima y la autonomía funcional de los ancianos hospitalizados. Motricidade, 2007;3(1):279-88.

11. American Thoracic Society/European Respiratory Society. ATS/ERS Statement on respiratory muscle testing. Am J Respir Crit Care Med. 2002;166:518-624.

12. American Thoracic Society and European Respiratory Society. Statement on Pulmonary Rehabilitation. Am J Respir Crit Care Med 2006:173(12):1390-413.

13. Pita-Fernández S, Pértega-Díaz S, Rodríguez-Maseda E. La fiabilidad de las mediciones clínicas: el análisis de concordancia para variables numéricas. Cad Aten Primaria 2003;10(4):290-6.

14. Szklo M, Nieto FJ. Epidemiología intermedia: conceptos y aplicaciones. Madrid: Ediciones Díaz de Santos; 2003.

15. González-Montesinos JL, Vaz Pardal C, Fernández Santos JR, Arnedillo-Muñoz A, Costa Sepúlveda JL, Gómez-Espinosa de los Monteros R. Efectos del entrenamiento de la musculatura respiratoria sobre el rendimiento. Rev Andal Med Deporte. 2012;5(4)163-70. doi:10.1016/S1888-7546(12)70025-4

16. Dimitriadis Z, Kapreli E, Konstantinidou I, Oldham J, Strimpakos N. Test/retest reliability of maximum mouth pressure measurements with the MicroRPM in healthy volunteers. Respir Care. 2011;56(6):77682. doi: $10.4187 /$ respcare. 00783

17. Montemezzo D, Velloso M, Britto RR, Parreira VF. Pressões respiratórias máximas: equipamentos e procedimentos usados por fisioterapeutas brasileiros. Fisioter Pesq. 2010;17(2):147-52. 
Wilches-Luna EC, Sandoval LM, López DJ

18. Bland JM, Altman DG. Comparing methods of measurement: why plotting difference against standard method is misleading. The Lancet 1995;346(8982):1085-7.

19. Lluís-Carrasco J, Jover L. Métodos estadísticos para evaluar la concordancia. Med Clin (Barc) 2004;122(Supl 1):28-34.

20. Parreira VF, França DC, Zampa CC, Fonseca MM, Tomich GM, Britto RR. Pressões respiratórias máximas: valores encontrados e preditos em indivíduos saudáveis. Rev Bras Fisioter 2007;(11):361-68.

21. Rodrigues F, Bárbara C. Pressões respiratórias máximas: proposta de um protocolo de procedimentos. Rev Port Pneumol. 2000;6(4):297-307. 